\title{
Decompaction and fluidization of a saturated and confined granular medium by injection of a viscous liquid or gas
}

\author{
Ø. Johnsen, ${ }^{1,2,3}$ C. Chevalier, ${ }^{1,4}$ A. Lindner, ${ }^{1}$ R. Toussaint, ${ }^{3}$ E. Clément, ${ }^{1}$ K. J. Måløy, ${ }^{2}$ E. G. Flekkøy, ${ }^{2}$ and J. Schmittbuhl ${ }^{3}$ \\ ${ }^{1}$ Laboratoire de Physique et Mécanique des Milieux Hétégogènes (PMMH), UMR 7636 CNRS, ESPCI, Universités Paris 6 et 7, 10, \\ rue Vauquelin, 75231 Paris Cedex 05, France \\ ${ }^{2}$ Advanced Materials and Complex Systems (AMCS), Department of Physics, University of Oslo, P. O. Box 1048 Blindern, \\ 0316 Oslo, Norway \\ ${ }^{3}$ Institute of Globe Physics in Strasbourg (IPGS), UMR 7516 CNRS, Université Louis Pasteur, 5 rue Descartes, \\ F-67084 Strasbourg Cedex, France \\ ${ }^{4}$ Université Paris Est, LCPC, MSRGI, 58 boulevard Lefebvre, 75015 Paris, France
}

(Received 19 June 2008; published 6 November 2008)

\begin{abstract}
We compare quantitatively two experimental situations concerning injection of a miscible fluid into an initially jammed granular medium saturated with the same fluid, confined in a Hele-Shaw cell. The two experiments are identical, apart from the interstitial and injected fluid, which is in one case air injected into a dry granular packing, and in the other case silicone oil injected into a dense suspension. In spite of the strong differences regarding the nature of the two fluids, strikingly similar dynamical and geometrical features are identified as functions of the control parameters: cell thickness and applied fluid injection pressure. In both cases an initial hydrodynamically driven decompaction process controls the unjamming and prepares the final displacement process characterized by fingerlike patterns empty of grains. The pattern shapes are comparable. In addition, the mobilities of the coupled fluid-grain flow, rescaled by the interstitial fluid viscosity and grain diameter squared, are of the same range and behave comparably. The mobility proves to depend on the initial solid fraction of the medium. Subtle differences are observed in geometrical aspects like the finger width with respect to the control parameters.
\end{abstract}

DOI: 10.1103/PhysRevE.78.051302

PACS number(s): 45.70.Qj, 47.15.gp, 47.55.Kf

\section{INTRODUCTION}

The hydrodynamic instabilities and fingerlike patterns emerging as a less viscous fluid displaces a more viscous fluid in Hele-Shaw cell configurations [1] have been extensively studied and vastly documented for both Newtonian [2-5] and non-Newtonian fluids [6-11]. Another flow situation with a well-established physical description of its own is that of a fluid flow through a fixed porous medium [12]. However, where these two systems merge, the miscible case of fluid injection into a deformable porous phase close to the jamming transition [13-18], the solid grains can behave as a conducting porous matrix, or can be suspended, forming an effective fluid. The transition between the two flow types has been studied during fluid injection into a loosely packed granular medium $[19,20]$ or a dense granular suspension [21], and is similarly observed when a fluid is retracted through a granular matrix $[22,23]$. The fluid displacement of grains by injection is an unstable process leading to the formation of fingerlike patterns $[19,20,24,25]$, visually quite similar to those found for non-Newtonian fluids. Coupled fluid-grain flows and fluid-grain interactions, on which these processes rely, are rather poorly understood and their study is in its infancy. The case of bubbling instability in fluidized beds under gravity is perhaps the most studied issue related to this case [26-37]. Nevertheless, for a very dense granular medium the situation is quite complex, essentially due to the nature of a granular material: it may act as a complex fluid and can jam at higher concentrations and become a solid porous matrix.

In practice, the problem addressed is an important issue since many industrial or geophysical applications rely on such an elementary process. Internal piping erosion due to flow concentration into tubular macropores during runoff may ultimately cause failure of soils in, e.g., dikes, embankments, and dams [38]. In nature, this type of erosion may also trigger hill-slope instabilities $[39,40]$, as well as sudden and potentially catastrophic drainage of the water contained in calderas [41], or in natural lakes formed after earthquakes.

In the present paper we present two experiments developed in parallel due to common interest in the following situation: injection of a fluid into a loosely consolidated granular packing with a solid fraction brought close to the random close packed (RCP) limit [42]. We study the fluidization process of an initially jammed granular medium.

Two simple model realizations of the miscible injection problem are considered: the granular material, consisting of monodisperse polystyrene spheres, is confined in a horizontal Hele-Shaw cell, ensuring identical situations as far as geometry is concerned. The injection is made with either air into the initially densely packed and dry assembly of spheres, or by a silicone oil invading a dense suspension made with the same granular material and the same silicone oil as injected. At sufficiently high overpressures, the drag exerted by the permeating fluid over the weakly consolidated granular medium mobilizes the grains. The goal of this study is to classify and describe quantitatively the mobilization of the initially jammed granular medium and the patterns emerging during this fluidization process, and comparatively reveal similarities and differences due to the nature of the surrounding and injected fluid.

The paper is organized as follows. First, in Sec. II the experimental setup and the principle of the injection experi- 


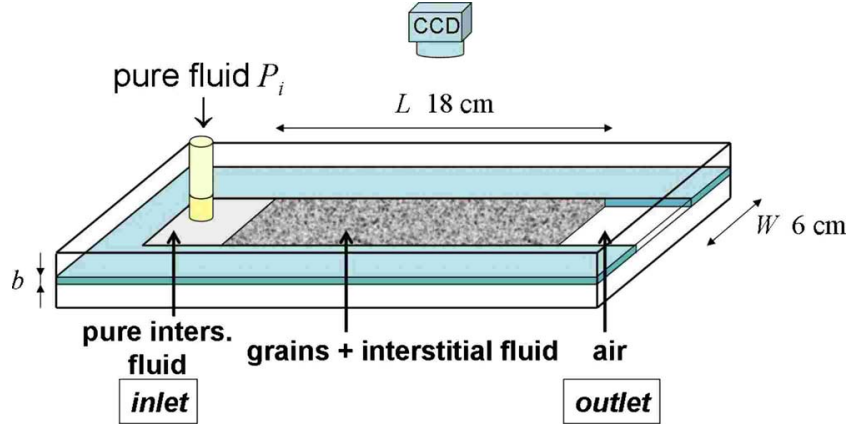

FIG. 1. (Color online) Experimental setup. A closed channel is made between two glass plates with three impermeable side boundaries and one open (right). Either air or silicone oil is injected at pressure $P_{i}$ into, respectively, a dry assembly of grains, or grains saturated with the same type of silicone oil. Around the inlet (left part of the cell) there is a grain-free zone occupied by pure fluid. The region ahead of the grain and fluid and up to the outlet (far right) is occupied by air at atmospheric pressure for both cases.

ments are described. Then in Sec. III the aim is to characterize the evolution of the unjamming process of typical experiments: decompaction dynamics and its role on the subsequent selection of the pure fluid fingerlike patterns emerging in the dense matrix. The flow properties and geometrical features of these fingerlike patterns are discussed in Sec. IV, before we summarize and address final conclusions in Sec. V.

\section{EXPERIMENTAL SETUP}

The experiments are performed in a linear Hele-Shaw cell [1] as illustrated in Fig. 1. A straight channel of rectangular section with a width $W=6 \mathrm{~cm}$ and a length of $L=30 \mathrm{~cm}$ is cut out of a Mylar sheet of calibrated thickness. The cell thickness is $b=0.85,1.15,1.70$, or $2.30 \mathrm{~mm}$. The Mylar sheet is sandwiched between two thick glass plates acting as spacers and ensuring that three of the sides of the channel are impermeable. The end of the channel is left open and will be referred to as the outlet. Opposite to the outlet there is an injection point $(\varnothing=7 \mathrm{~mm})$ in the upper plate positioned $1 \mathrm{~cm}$ from the impermeable boundary, referred to as the inlet.

The granular medium in the cell is saturated with a given interstitial fluid as described in Secs. II A and II B. In this work, we consider two cases with clearly distinct fluids: air (compressible, viscosity $\eta_{\text {air }}=1.8 \times 10^{-5} \mathrm{~Pa} \mathrm{~s}$ ), and silicone oil (incompressible, viscosity $\eta_{\text {oil }}=5.0 \times 10^{-3} \mathrm{~Pa} \mathrm{~s}$ ). The space around the inlet is initially filled with the same fluid as the interstitial one. This space acts as a pressure chamber. At the beginning of the experiment, the fluid injection starts at a given injection pressure $P_{i}=\Delta P+P_{\mathrm{atm}} . \Delta P$ is the fluid overpressure with respect to atmospheric pressure $P_{\text {atm }}$, at the outlet. $\Delta P$ is applied by manually opening a valve. Opening the valve fully takes $\sim 0.02 \mathrm{~s}$. Thus, the pressure profile of the injection is close to a step function: after the onset, $\Delta P$ is maintained constant throughout the experiment. The probed range of the applied $\Delta P$ is between $\sim 10$ and $\sim 200$ mbars for the dense packing prepared by tapping.
Complementary to the comparative study involving two distinct fluids for otherwise similar conditions, we have performed experiments with an initially less dense packing of the granular material. In this case, the cell thickness is $b$ $=0.70,1.10$, and $2.1 \mathrm{~mm}$, and $\Delta P$ is in the range $\sim 2$ to $\sim 30$ mbars.

To record the motion of the grains, images are obtained by reflection, with the use of a dark background to increase the contrast between the zones free of grains and the granular regions. The setup is illuminated from above using two $800 \mathrm{~W}$ halogen projectors, and images of $400 \times 1600$ pixels are acquired at a frequency of 500 images per second, using a Phantom V9 high-speed camera for the air-grain experiments, and for the oil-grain system a Prosilca EC1280 charge-coupled device (CCD) camera is used at a sample frequency of ten images per second, at a resolution of $1280 \times 400$ pixels.

\section{A. Sample preparations: Air-grain system}

The granular medium consists of spherical monodisperse polystyrene beads (Dynoseeds) with a diameter $d$ $=80 \pm 3 \mu \mathrm{m}$, and density $\rho_{\text {grain }}=1.05 \pm 0.01 \mathrm{~g} \mathrm{~cm}^{-3}$. To ensure a good reproducibility of the experiments, the following preparation stages were adopted to put the granular medium into place: the cell is placed vertically and the grains are introduced via the outlet. Once the proper quantity is introduced, a Mylar plate matching the width and height of the channel is inserted $\sim 6 \mathrm{~cm}$ into the channel. This will prevent the grains from moving through the outlet during the sample preparation. The cell is then tilted upside down, which brings the granular medium against the Mylar plate, and frees space around the inlet. The granular material is further compacted by tapping the filled cell in a defined manner, which ultimately brings the packing into a dense initial state with solid fraction $\rho_{0}=(62 \pm 1) \%$, found by weighing the grains and measuring the volume they occupy in the cell. This is close to the random close packed limit [42].

For the complementary experiments the procedure is the same, except that in this case the particles are simply assembled in the cell under gravity without mechanically imposing any further compaction. The solid fraction is $\rho_{0}$ $=(56 \pm 1) \%$.

During the sample preparation solid contacts between the grains are established and friction forces from the walls on the grains are mobilized, pointing toward the inlet. The preparation stages produce a granular sample with a length $L_{0}=18 \mathrm{~cm}$. The grain-free zone around the inlet is about $\sim 6 \mathrm{~cm}$ and the air-grain boundary facing the inlet is reasonably straight. This interface is referred to as the inner boundary. The cell is then brought into a horizontal position and the Mylar plate used during the preparation is removed, leaving a straight grain-air boundary facing the outlet. We refer to this boundary as the outer boundary.

For the experiments performed with air as the interstitial fluid, control of the humidity of the experimental environment during sample preparations is important. The interactions between $\sim 80-\mu \mathrm{m}$-sized beads are sensitive to the humidity of the surroundings: in very dry conditions, 


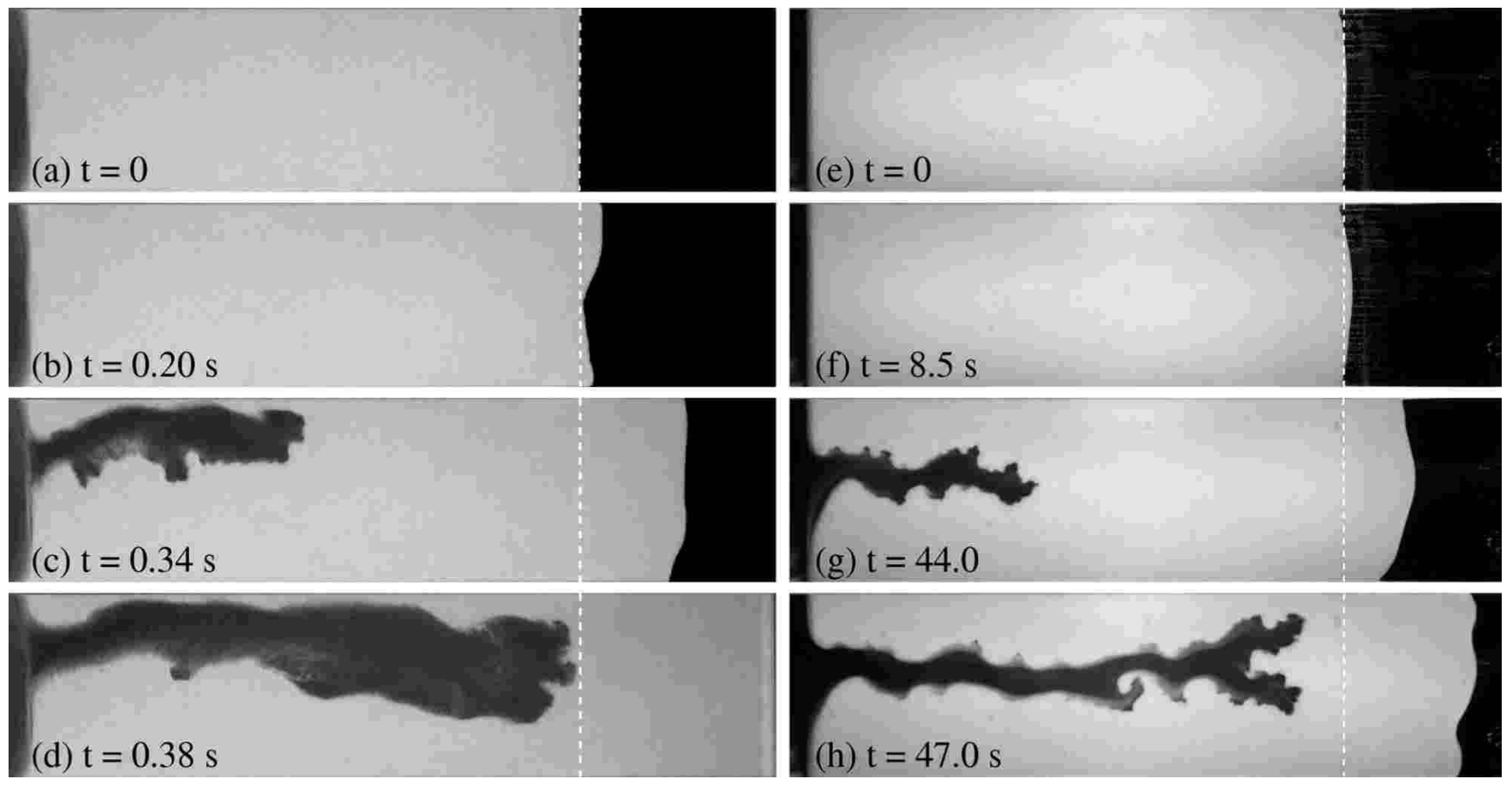

FIG. 2. Typical experiments. The left column shows an air-grain experiment at $\Delta P_{\text {air }}=93 \mathrm{mbar}$ and $b=1.15 \mathrm{~mm}$. In the right column an oil-grain experiment at $\Delta P_{\mathrm{oil}}=85 \mathrm{mbar}$ and with $b=1.15 \mathrm{~mm}$ is displayed. (a) and (e) shows the sample before fluid injection. The dashed lines indicate the initial position of the outer boundary, $L_{0}$. Before a finger empty of grains starts developing the granular packing has been dilated, indicated by an increase of length beyond $L_{0}$ seen in (b) and (f). In (c) and (g) typical finger patterns are shown, after propagating for $L_{0} / 2$, and in (d) and (h) the fingers have propagated a distance $L_{0}$.

electrostatic forces play a role, while under excessively humid conditions, capillary bridges form between neighboring beads, resulting in small but significant cohesive forces. To ensure the reproducibility of these individual interactions, the relative humidity of the air was kept between $30 \%$ and $40 \%$.

\section{B. Oil-grain system}

For the oil-grain system the same beads and cells are used. An identical sample preparation procedure to obtain the dense packing for the air-grain is applied. But before removing the Mylar plate that blocks the outlet a different final step is performed: imbibition of the granular medium with the silicone oil. Note that, in order to imbibe the medium successfully without trapping air bubbles and to avoid rearrangement of grains leading to large density heterogeneities within the granular matrix, the packing fraction must be near the close packed limit. Hence, only the dense initial packing is obtainable for the oil-grain case.

The granular medium is imbibed with a silicone oil Rhodorsil 47V05 from Rhodia Silicones with a viscosity $\eta_{\text {oil }}=5.0 \times 10^{-3} \mathrm{~Pa} \mathrm{~s}$, as given by the supplier and measured with a Thermo-Haake rheometer. The oil is introduced via the inlet from an oil reservoir at constant overpressure. During imbibition of oil into the granular medium one can observe a propagating imbibition front. (Note that the Mylar plug prevents the grains from moving and oil from escaping but is permeable to air.) The imbibition front is a straight line, indicating a homogeneous porosity of the dry jammed grains and validating our preparation step. When measuring the velocity of the imbibition front as a function of the applied pressure gradient, one can deduce the permeability of the porous medium [12]. Applying the Carman-Kozeny relation [43] the solid fraction estimation is $\rho_{0} \sim 60 \%$, which agrees with the solid fraction obtained by weighing the grains and measuring the occupied volume of the granular medium. Once the porous medium is completely immersed the pressure is reduced to atmospheric pressure (corresponding to a zero pressure gradient) and the Mylar plug is removed. The initial state consists then of a zone of grains fully immersed in a silicone oil and limited by two welldefined interfaces. The interface between the pure oil and the immersed granular medium facing the inlet is referred to as the inner boundary and the interface between the granular medium and air, facing the outlet, is referred to as the outer boundary. Note that an oil-grain-air interface at the outer boundary is chosen out of reproducibility considerations. This leads to a difference in the boundary conditions by the presence of interfacial surface tension. The oil and the grains are close to density matched; thus, sedimentation can be neglected.

\section{EMERGING PATTERNS}

\section{A. Typical experiments}

Figures 2(a) and 2(e) show images respectively of the air-grain and the oil-grain systems just before injection (at $t_{0}$ ) for cell thickness $b=1.15 \mathrm{~mm}$, at a comparable $\sim 60 \%$ initial 
solid fraction. When a $\Delta P$ within the given pressure range is applied at the inlet, no grain motion is detected along the inner boundary at the onset of the fluid flow. Moving grains are first observed on the outer boundary that starts to deform, typically appearing slightly bulged. This decompaction stage is visible in Figs. 2(b) and 2(f). The decompaction process deserves more attention and will be described in depth in the next section. While the outer boundary progresses the total grain-fluid area increases, indicating an average decrease of solid fraction, i.e., the medium is decompacted. During such a process, the friction forces between the grains and the confining plates are decreased, and the grains start to move with the interstitial fluid. After a characteristic time, the grains are mobilized over the entire length of the packing and the inner boundary starts to move. The inner boundary rapidly deforms and departs from the initial straight shape. A finger empty of grains forms in the center of the cell, signaling an unstable penetration mechanism. The shape of this finger is illustrated in Figs. 2(c) and 2(d) for the air-grain system, and in Figs. 2(g) and 2(h) for the oil-grain case. In Figs. 2(c) and $2(\mathrm{~g})$ the finger tip has propagated a distance $L_{0} / 2$, and in Figs. 2(d) and 2(h) a distance $L_{0}$, respectively the half and the full extent of the initial system length. The similar aspect of this finger between the air-grain and the oil-grain systems is visible qualitatively on these images. They are also reminiscent of fingerlike structures observed in many nonNewtonian fluids [6-11].

This behavior is systematically observed in the oil-grain system, for the probed range of $\Delta P$ (from 10 up to 200 mbars). On the other hand, for the air-grain system, there is a threshold to exceed for the applied $\Delta P$ in order to set the grains into motion from the jammed state. For $\Delta P$ below this threshold there is no observed deformation of the outer grain-air boundary and no finger propagation: the air permeates through the grains without demobilizing the friction forces. The threshold is dependent on cell thickness $b$, as shown in Fig. 3. The threshold is also probed by gradually increasing the fluid overpressure from zero: the gray square markers indicate at which $\Delta P$ the fluidization and the finger patterns first take place during this pressure ramp. A threshold for the oil-grain case is likely, but might be located at a lower $\Delta P$ than our pressure control device allows. A contingent absence is another possibility, and might in that case rely on several factors which are difficult to assess, given the sample preparation procedure. Hypothetically, the presence of an interface with surface tension might pull particles along when the fluid flow is initiated; in addition, lubrication forces and the low level of buoyancy forces might alter the friction conditions between the granular matrix and the confining walls.

Eventually, we notice that the deformation of the outer boundary starts after a short delay after the injection for the air-grain system, whereas no delay is observed for the oilgrain system. This delay has also been observed in experiments performed in larger cells with a similar air-grain system [20] and has been shown to originate from the finite compressibility of the interstitial fluid. It corresponds to a diffusive propagation of the overpressure through the packing. The characteristic time for this diffusion is $L_{0}^{2} / D$, where the diffusivity constant is $D=\kappa_{0} /\left(\eta_{\text {air }} C\right) . \kappa_{0}=5.1 \times 10^{-12} \mathrm{~m}^{2}$

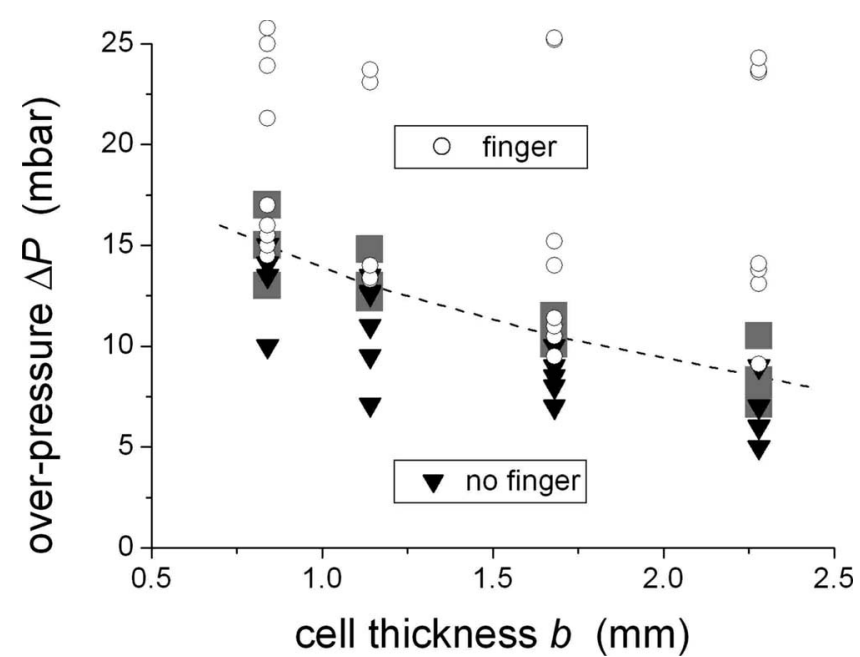

FIG. 3. Threshold pressure for the onset of grain mobilization and finger formation in the air-grain system. The gray squares correspond to the $\Delta P$ at which fingering occurs for experiments where the injection pressure is gradually increased from zero. For the oilgrains system finger formation is observed for all tested $\Delta P$.

is the permeability of the granular packing at the initial solid fraction $\rho_{0} \sim 62 \%$, and is estimated by the Carman-Kozeny relation [43]. $\eta_{\text {air }}$ is the viscosity of the interstitial fluid, and $C$ is the compressibility of the fluid $[20,26]$. The pressure diffusivity for air is found to be $D \simeq 0.04 \mathrm{~m}^{2} / \mathrm{s}$, so that for the small cells considered here with $L_{0} \simeq 0.18 \mathrm{~m}$, the characteristic time is around $1 \mathrm{~s}$. This delay is observable in the experiments, but is very short with respect to the total duration of the experiment. For the system with grains and oil, the oil's vanishing compressibility makes this time much too small to be observable.

The global dynamic behavior of the dense air-grain and oil-grain systems is significantly different from that of the complementary air-grain experiments with a lower initial solid fraction. No contraction of the granular matrix leading to a compaction front traveling in the fluid flow direction starting from the inner boundary is observed, as in the case of an initially loose packed granular material [20].

\section{B. Decompaction}

During the initial stage, while the outer boundary moves and the inner one stays fixed, we analyze the internal decompaction by applying an image subtraction treatment. This post-treatment will allow us to resolve spatially the structural features of the decompaction process. An image shot before applying the overpressure $\Delta P$ serves as a reference frame. Its pixel gray-level map (numbers between 1 and 256, from dark to bright) is subtracted from the pixel gray-level values of an image at time $t$ during the fluid injection. The absolute values of these differences form a gray-level map which reflects the particle mobilization relative to the reference image: the mobile zones appear bright, while the immobile zones appear dark. To further increase the contrast between the mobile and immobile zones the resulting image difference is filtered by applying a threshold determined by a local minimum be- 
tween the two main peaks in the gray-level histogram of individual images. The image is then binarized by putting all pixels with a value below this threshold to 1 and all pixels with a value exceeding the threshold to 256 . The finger structures appear as solid white, but also very small particle displacements can be detected and recognized as white speckles. The decompacted region is identified as a clearly defined zone of high speckle density. To separate the densely speckled region from speckles that originate from, e.g., noise due to small light intensity variations, we check for each speckle the nearest neighbors within a range of $\sim 4 \times 4 \mathrm{~mm}^{2}$. If two or more speckles are contained within this range, the grain area within a spanned rectangle defined with the speckles as the vertices is tagged in gray. Figures 4(c) and 4(d) show the treated images for the two sets of experiments shown in Figs. 4(a) and 4(b).

The decompaction process does not correspond to a homogeneous decrease of the solid fraction over the whole granular material, but to a localized decompaction zone starting from the outer boundary. The decompacted zone is a region of mobile grains carried with the flowing fluid in an area $A_{\mathrm{dec}}(t)$ determined by analysis of the treated images. When the initial packing fraction $\rho_{0}$ is known a priori, we estimate the average solid fraction $\rho_{\mathrm{dec}} \sim 59 \%$ for both of the systems. Hence, the relative decompaction of the air-grain system is slightly larger than for the oil-grain system, with their respective initial packing fractions of $\rho_{0} \sim 62 \%$ and $\sim 60 \%$ (evident in Fig. 7). As a contrast, in the immobile zone the fluid permeates through grains that stay fixed. A boundary between the immobile and the decompacted zone can thus be distinguished as a decompaction front. The solid friction between the grains and the wall gets demobilized, and the granular medium gets unjammed when the front passes. The decompaction front moves backward, opposite to the average fluid flow, toward the inlet of the channel.

In both the oil-grain and air-grain systems, the mobile zone growing from the outer boundary evolves into fingerlike structures. It is worth noticing that several fingers may form and progress in parallel (see Fig. 4). In the air-grain system, as many as four decompaction fingers were observed during the initial stage of injection. The decompaction fingers observed in the oil-grain system are typically wider.

\section{Waiting time}

The waiting time denoted $t_{w}$ is defined as the time elapsed from the moment when grain motion is first observed at the outer boundary until a propagating finger structure is initiated at the inner boundary. It is the typical time for the decompaction front to move over the whole system length $L_{0}$. Since the decompaction process is a direct result of the drag force exerted on the particles, the fluidization process is expected to occur after shorter $t_{w}$ with increasing $\Delta P$. This is indeed confirmed in Fig. 5. On the other hand, there is no clear dependence on the cell thickness $b$, except for the airgrain system where the experiments performed with $b$ $=2.3 \mathrm{~mm}$ distinguish themselves. In this case, the difference might be attributed to the role of gravity and decreased role of friction as one crosses over to a more three-dimensional packing ( $b=2.3 \mathrm{~mm} \sim 29$ particle diameters). This is not an issue in the oil-grain system since we have an isodense suspension.

By recognizing two zones with distinguishable solid fractions, we construct a simple analytical model for the waiting time, mainly based on mass conservation. For simplicity, we consider a one-dimensional model in the sense that the decompaction front is flat and uniform over the cell width, and perpendicular to the sidewalls (see Fig. 6). With the inner boundary defined as $X=0$, the initial condition is $X_{g}(t=0)$ $=X_{\mathrm{dec}}(t=0)=L_{0}$. Here $X_{g}$ is the position of the outer boundary, $X_{\mathrm{dec}}$ is the position of the decompaction front, and $L_{0}$ is the initial length of the packing. The length of the decompaction zone at a time $t$ is $l_{\mathrm{dec}}(t)=X_{g}(t)-X_{\mathrm{dec}}(t)$. At an arbitrary time $t$, conservation of mass and grains gives

$$
\rho_{0} X_{\mathrm{dec}}+\rho_{\mathrm{dec}} l_{\mathrm{dec}}=\rho_{0} L_{0} .
$$

Conservation of grain flux links the velocity of the outer boundary $V_{g}=d X_{g} / d t$, and the velocity of the decompaction front $V_{\mathrm{dec}}=d X_{\mathrm{dec}} / d t$, through

$$
V_{g}=\left(1-\frac{\rho_{0}}{\rho_{\mathrm{dec}}}\right) V_{\mathrm{dec}} .
$$

For the oil-grain case, the fluid velocity into the decompacted region equals the velocity of the outer boundary, and the Darcy law gives

$$
\frac{P_{i}-P_{\mathrm{dec}}}{X_{\mathrm{dec}}}=\frac{\eta_{0}}{\kappa_{0}\left(\rho_{0}\right)} V_{g} .
$$

$P_{i}-P_{\text {dec }}$ is the pressure drop over the nondecompacted material, which acts as a fixed porous material with a CarmanKozeny permeability $\kappa_{0}\left(\rho_{0}\right) . P_{i}$ is the injection pressure, and $P_{\mathrm{dec}}$ is the pressure at the front positioned at $X_{\mathrm{dec}}$. Similarly, over the decompacted region Darcy's relation gives

$$
\frac{P_{\mathrm{dec}}-P_{\mathrm{atm}}}{l_{\mathrm{dec}}}=\frac{\eta_{\mathrm{paste}}}{\kappa_{\mathrm{HS}}(b)} V_{g} .
$$

Here the grains and the fluid are considered as an effective fluid with a viscosity $\eta_{\text {paste }}$, and the permeability is given by the confining space of the Hele-Shaw cell as $\kappa_{\mathrm{HS}}(b)=b^{2} / 12$. We introduce "paste" as a common nomenclature for both the air-grain and the oil-grain systems, referring to the granular material and the fluid flowing together.

The above flux conservation consideration is valid only if the fluid velocity is comparable to the velocity of the outer boundary. For the oil-grain system the fluid-air interface adjacent to the outlet is visually accessible, and the fluid flux is measurable. The velocity of the outer grain boundary, $V_{g}$, is the same as the oil velocity $V_{\text {oil }}$. Since the fluid is not visually traceable and flux measurements not made available for the air-grain case, we estimate the fluid seepage velocity relative to the grains, $V_{\text {flux }}$, using the Darcy relation. At the instant just before forming a finger, the granular medium is decompacted over the whole system length. The CarmanKozeny permeability is $\kappa\left(\rho_{\mathrm{dec}}=59 \%\right)=7.0 \times 10^{-12} \mathrm{~m}^{2}$, and $V_{g}$ is typically from 0.5 to $20 \mathrm{~cm} \mathrm{~s}^{-1}$ for the corresponding $\Delta P$ from 10 to 200 mbars. With the fraction 

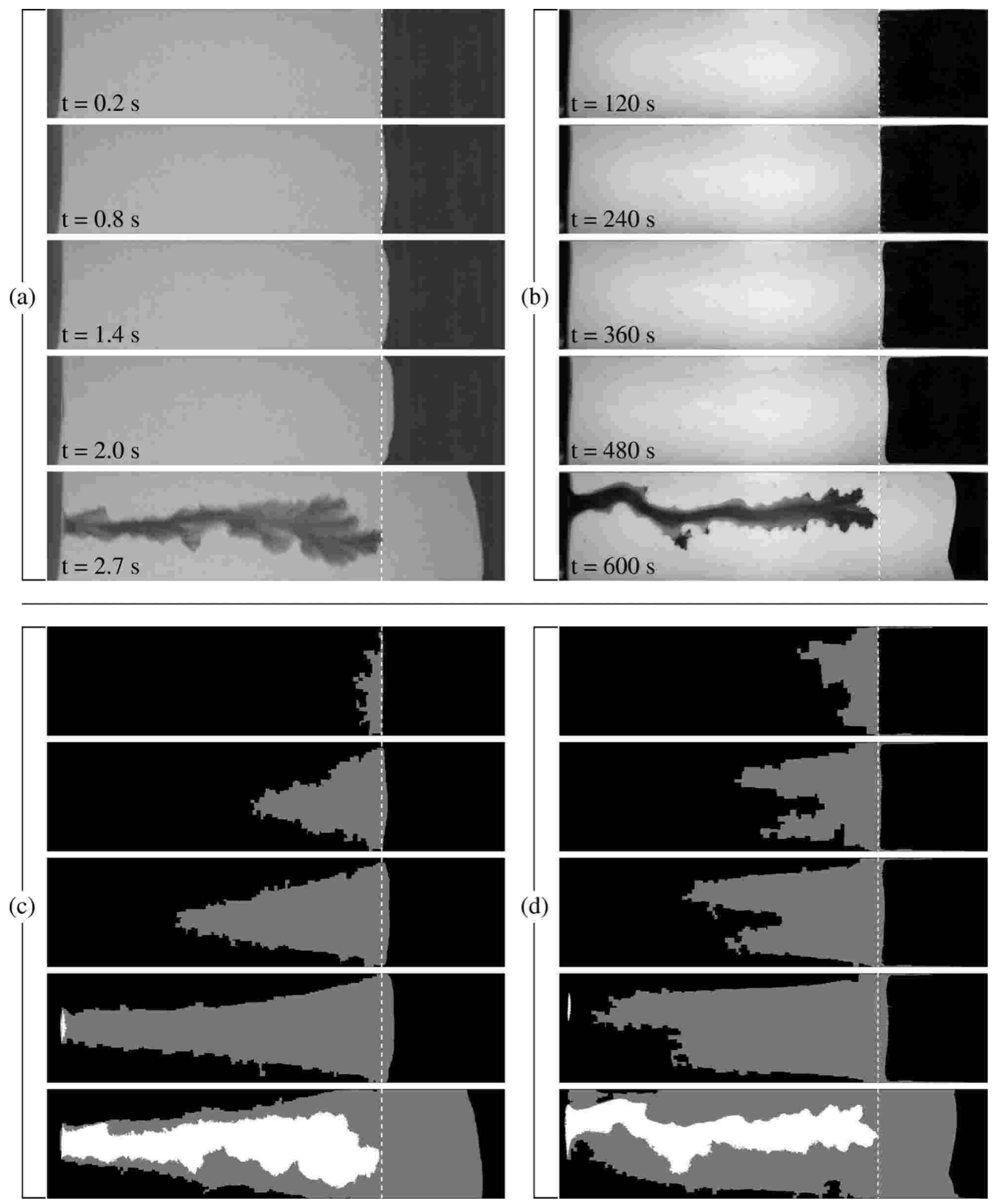

\section{\begin{tabular}{l|ll} 
Immobile zones & Mobile/decompacted zone & Emptied region
\end{tabular}}

FIG. 4. Typical experiments. Images in (a) and (b) show the raw images for, respectively, air-grain and oil-grain mixtures, while in (c) and (d) the corresponding subtracted and filtered images show the mobile zones. Air-grain system: $b=1.15 \mathrm{~mm}, \Delta P=13 \mathrm{mbars}$. Oil-grain system: $b=1.15 \mathrm{~mm}, \Delta P=11 \mathrm{mbars}$. The dashed vertical lines indicate the initial position of the outer grain boundary. The bar on the bottom displays the color code representing immobile zones with solid fraction $\rho_{0}$, mobile or decompacted zone with solid fraction $\rho_{\text {dec }}<\rho_{0}$, and the finger virtually emptied of grains where $\rho \sim 0$. 


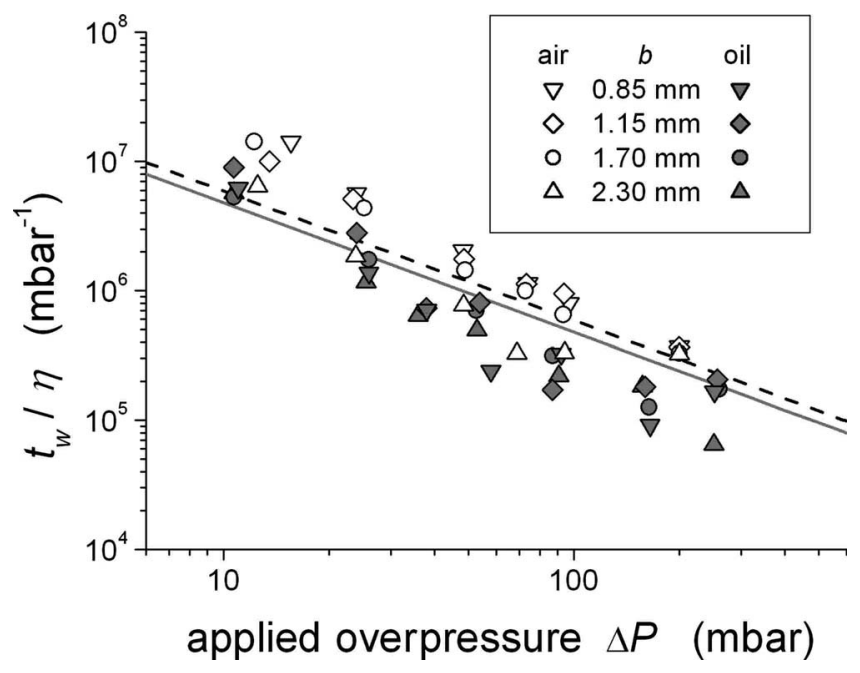

FIG. 5. Log-log representation of the waiting time $t_{w}$ scaled with the fluid viscosity, as a function of the applied $\Delta P$. The dashed line is the modeled waiting time for the air-grain system, and the solid line is the modeled waiting time for the oil-grain case.

$$
\frac{V_{\text {flux }}}{V_{g}}=\frac{\left(\kappa / \eta_{\text {air }}\right) \nabla P}{V_{g}} \sim 0.43,
$$

the proposed model seems reasonable to use also for the air-grain system.

Combining Eqs. (3) and (4), we obtain

$$
\frac{P_{i}-P_{0}}{V_{g}}=\frac{\eta_{0}}{\kappa_{0}} X_{\mathrm{dec}}+\frac{\eta_{\text {paste }}}{\kappa_{\mathrm{HS}}} l_{\mathrm{dec}} .
$$

From Eq. (1) we have $l_{\mathrm{dec}}=\rho_{0} / \rho_{\mathrm{dec}}\left(L_{0}-X_{\mathrm{dec}}\right)$, and by performing a substitution of $V_{g}$ using Eq. (2), Eq. (6) yields

$$
\frac{\Delta P}{V_{\mathrm{dec}}}=\left(1-\frac{1}{c}\right)\left[\frac{\eta_{\text {paste }}}{\kappa_{\mathrm{HS}}} \frac{1}{c} L_{0}+\left(\frac{\eta_{0}}{\kappa_{0}}-\frac{\eta_{\text {paste }}}{\kappa_{\mathrm{HS}}} \frac{1}{c}\right) X_{\mathrm{dec}}\right] \text {, }
$$

where $c=\rho_{\mathrm{dec}} / \rho_{0}$. By using $V_{\mathrm{dec}}=d X_{\mathrm{dec}} / d t$ and integrating with the boundary conditions $t=0, X_{d}=L_{0}$, and $t=t_{w}, X_{\mathrm{dec}}$ $=0$, we obtain the time $t_{w}$ for the decompaction front to

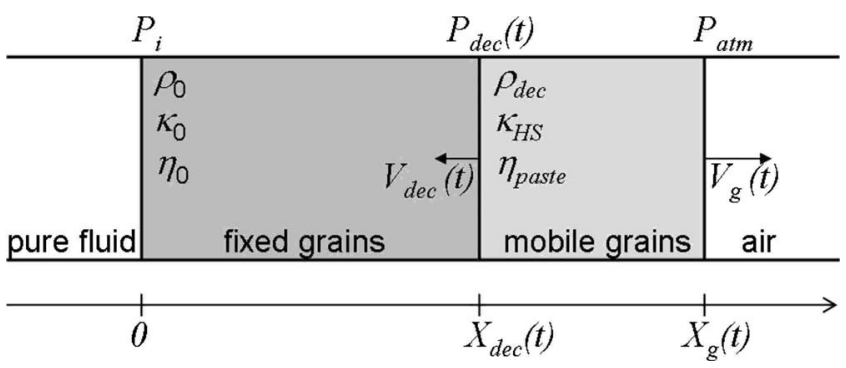

FIG. 6. Scheme and quantities involved in the modeling of the decompaction process.

travel the length of the granular packing. In the framework of this model, the waiting time becomes

$$
t_{w}=\frac{(1 / c-1) L_{0}^{2}}{2 \Delta P}\left(\frac{\eta_{\text {paste }}}{\kappa_{\mathrm{HS}}} \frac{1}{c}+\frac{\eta_{0}}{\kappa_{0}}\right) .
$$

We now determine the quantities of the model: $c$ is measured experimentally as $A_{0} / A(t)$, and gets its value from Fig. 7 at the instant $t=t_{w}: c_{\text {air }} \sim 0.985$ and $c_{\text {oil }} \sim 0.987$. The viscosity of the pure fluid $\eta_{0}$ is $1.8 \times 10^{-5} \mathrm{~Pa} \mathrm{~s}$ for air and 5.0 $\times 10^{-3} \mathrm{~Pa} \mathrm{~s}$ for the silicone oil. The initial permeability is estimated using the Carman-Kozeny relation giving $\kappa_{0}=5.1$ $\times 10^{-12} \mathrm{~m}^{2}$ at $\rho_{0} \sim 62 \%$ for the air-grain system, and for the oil-grain system $\kappa_{0}=6.6 \times 10^{-12} \mathrm{~m}^{2}$ at $\rho_{0} \sim 60 \%$. Analogously to flow of a simple fluid in a Hele-Shaw cell, Darcy's law is used in order to link $\eta_{\text {paste }} / \kappa_{\mathrm{HS}}$ to the applied pressure gradient and the flow velocity, via the mobility $M_{\text {paste }}^{\text {dec }}$ :

$$
M_{\text {paste }}^{\text {dec }}=\frac{\kappa_{\mathrm{HS}}}{\eta_{\text {paste }}}=\frac{V_{g}}{|\nabla P|},
$$

where $\nabla P=\Delta P / X_{g}(t)$ is the pressure gradient over the length of the granular packing $X_{g}\left(t=t_{w}\right) . V_{g}$ and $\nabla P$ are measured independently for each experiment at the instant $t=t_{w}$, which is just on the verge of finger propagation, when the packing has been decompacted over the whole length $L_{0}$. The paste mobility at $t_{w}$ is roughly $1.3 \times 10^{-6} \mathrm{~m}^{2} \mathrm{~Pa}^{-1} \mathrm{~s}^{-1}$ for the air-
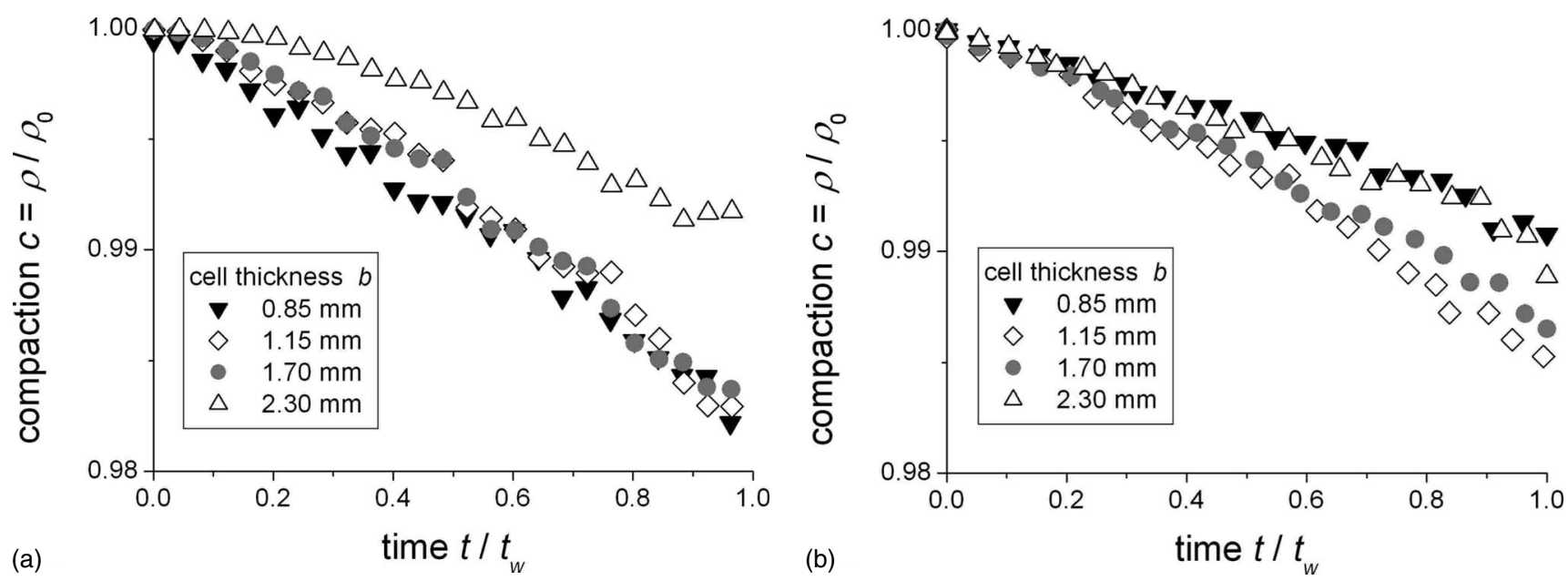

FIG. 7. Temporal evolution of the average global solid fractions $\rho(t) / \rho_{0}$ for (a) air-grain and (b) oil-grains systems. Along the $x$ axis the time $t$ is scaled with respect to the waiting time $t_{w}$. 


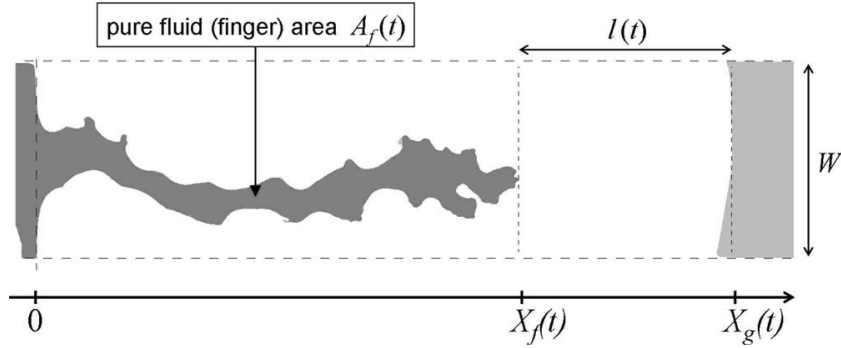

FIG. 8. Quantities measured during image analysis of the propagating fingers empty of grains.

grain system, and $2.8 \times 10^{-9} \mathrm{~m}^{2} \mathrm{~Pa}^{-1} \mathrm{~s}^{-1}$ for the oil-grain system.

The modeled waiting time scaled by the respective fluid viscosity is given in Fig. 5. Given the one-dimensional model, which represents a significant simplification of the observed decompaction front, and other simplifications like the assumed Darcy law for the mobile paste across the cell, the model compares favorably with the experiments. The $1 / \Delta P$ relation of modeled waiting time fits with the experimental data for the higher applied overpressures, while slightly deviating at lower pressures.

\section{IV. “EMPTY” FINGER PROPAGATION}

It is evident that the decompaction process prepares the medium for further channeling. The subsequent fingerlike structure empty of grains progresses preferentially in the zone that has been decompacted during the passage of the decompaction front. In this section we focus on the flow properties of the granular material and the finger structures developing after the unjamming process.

\section{A. Image analysis and definition of the measured quantities}

In order to quantitatively compare the two systems we identify the measurements of interest, as given in Fig. 8. Great effort has been put into numerically processing the subsequent images as closely as possible, so as to extract time-dependent quantities that can be compared.

The driving force of the grain motion is the pressure gradient $|\nabla P|$. It can be estimated as the pressure difference over the extent of the paste, $\Delta P / l(t)$, where $l(t)=X_{g}(t)-X_{f}(t)$ is the length of the paste ahead of the most advanced point of the finger. $X_{f}$ is the linear extent of the finger, and $X_{g}$ is the position of the outer air-grain boundary. $\Delta P$ is considered constant within the finger empty of grains. Due to a very high permeability contrast with that of the confined paste, the entire pressure drop is over the paste.

We take advantage of the mobility to characterize and quantify the coupled fluid-grain flow. In the same spirit as the definition of the paste mobility during the decompaction stage given in Eq. (9), the mobility during finger propagation is

$$
M=\frac{V_{\text {paste }}}{|\nabla P|} .
$$

We adapt a common practice among similar studies and determine the mobility by measuring the velocity of the paste pushed ahead of the propagating finger as

$$
V_{\text {paste }}=\frac{1}{W} \frac{d A_{f}(t)}{d t},
$$

where $A_{f}(t)$ is the area of the finger structure, and $W$ is the cell width. This definition of $V_{\text {paste }}$ comes from mass conservation and is effectively the same as $\lambda V_{f}$, where $V_{f}$ is the finger tip velocity, and $\lambda=w_{f} / W$ with $w_{f}$ as the average finger width. Note that, even though we are dealing with a miscible two-phase system, Eqs. (10) and (11) assume that $\nabla P$ is entirely driving the finger propagation, and seepage is negligible during this stage. We compare the typical velocities of the fluid flux into the pore space of the granular matrix $V_{\text {flux }}$, and the finger tip velocity $V_{f}$. The permeability $\kappa$ is between 5.1 and $7.0 \times 10^{-12} \mathrm{~m}^{2}$, and $V_{f}$ is typically $20-50 \mathrm{~cm} \mathrm{~s}^{-1}$ for the air-grain and $0.1-2 \mathrm{~cm} \mathrm{~s}^{-1}$ for the oilgrain system. Within the probed range of pressures, we find the fraction

$$
\frac{V_{\text {flux }}}{V_{f}}=\frac{\left(\kappa / \eta_{0}\right) \nabla P}{V_{f}}
$$

to be less than $10 \%$ for the air-grain and less than $1 \%$ for the oil-grain system. This suggests that the velocity of the fluid flow on the pore scale is negligible compared to the finger velocity, but comparable to the grain velocity during finger propagation. $\nabla P$ is thus mainly driving the finger and thus the paste, rather than contributing to seepage. This validates the use of Eqs. (10) and (11).

As the patterns are relatively complex, we choose the definition for the individual finger width $w_{i}=2 A / s$, where $A$ is the area occupied by the finger empty of grains divided by half of the perimeter $s$. We always consider the finger only over a distance of $6 \mathrm{~cm}$ (corresponding to the cell width $W$ ) measured from the finger tip. This approach of measuring the finger width corresponds to considering a single branched structure.

\section{B. Flow properties}

When the emptied finger propagates through the higherpermeability path prepared during the decompaction stage, the granular material is pushed in front of the finger tip. We use the mobility to characterize the flow properties and show to what extent the material is mobilized as a function of the applied overpressure $\Delta P$ and the cell thickness $b$.

Surprisingly, we find that, unlike flow of viscous fluids in Hele-Shaw cells and flow in porous media, the mobility is observed to be increasing during finger propagation, for a given experiment at constant $\Delta P$. The mobility is thus represented as a function of this finger tip position $X_{f}$, for a given $b$, and varying $\Delta P$, as in Figs. 9(a) and 9(b).

The almost linearly increasing mobility observed for the air-grain system as well as for the oil-grain system, implies that the granular material is increasingly fluidized and flows more easily during the course of the experiment. We cannot at the moment precisely account for the evolution of the mobility, and despite the strong similarities observed in the two systems, this phenomenon might originate from different mechanisms.

For the air-grain case, the evolving mobility might be attributed to the continuous decompaction of the granular ma- 

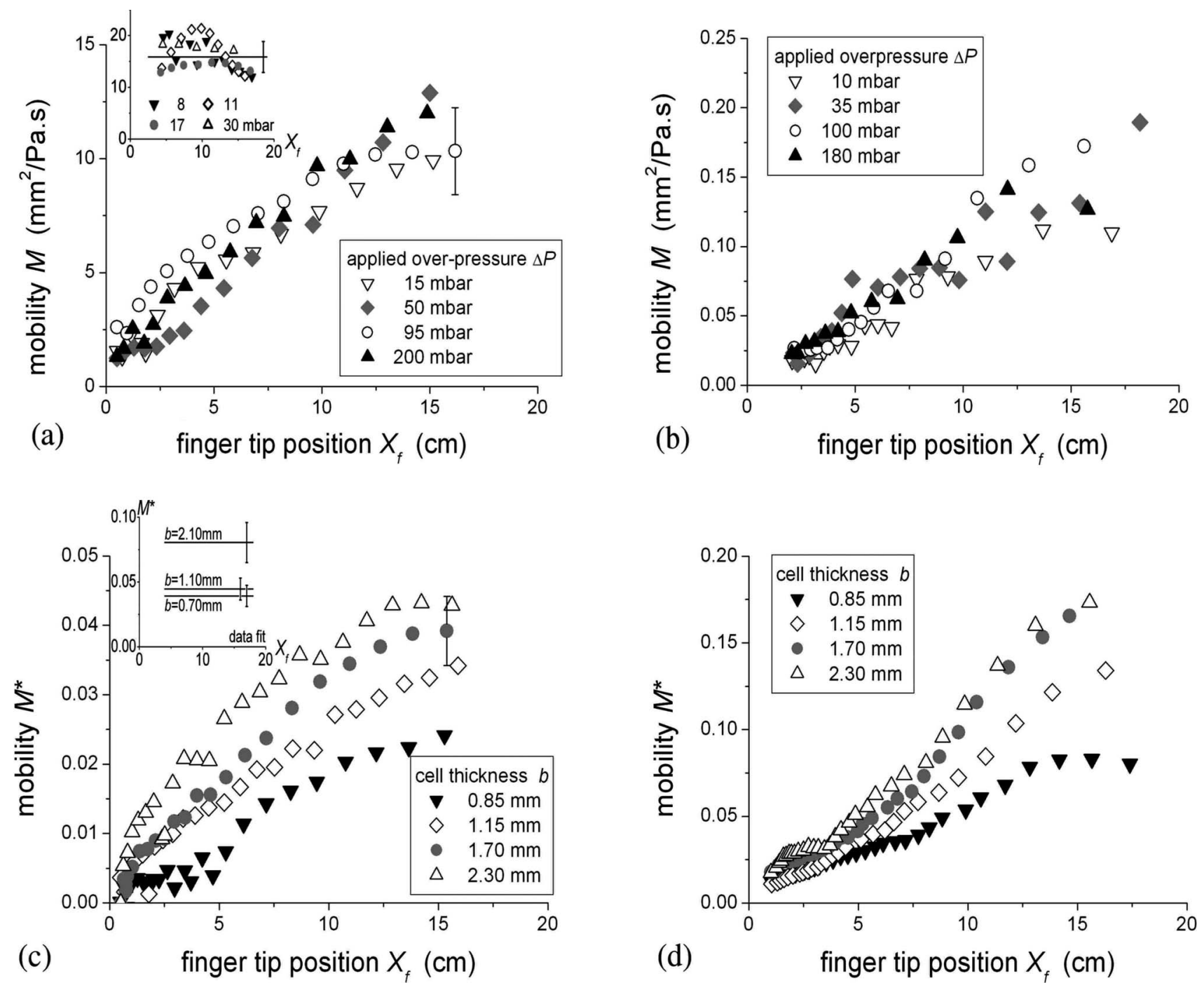

FIG. 9. Mobility $M$ as a function of finger tip position $X_{f}$ for (a) air-grain and (b) oil-grain systems. The cell thickness $b=1.15 \mathrm{~mm}$. (c) and (d) show the rescaled mobility $M^{*}=M /\left(d^{2} / \eta\right)$ as a function of finger tip position for air-grain and oil-grain systems, respectively. The rescaled mobility is averaged over all $\Delta P$ for each given $b$. Approximately 27 trials are used for the air-grain experiments and seven for the oil-grain case. The insets similarly show $M$ and $M^{*}$ obtained for the experiments involving an initially loosely packed granular matrix.

terial during finger propagation, as shown in Fig. 10. The solid fraction of decompacted material versus the initial fraction seems to be independent of the applied $\Delta P$. Note that in some cases it is not possible to reliably estimate the compaction index during finger propagation for the air-grain system. For experiments performed with a large cell thickness $b$ and a low $\Delta P$, particles tend not to be as effectively transported along with the air flow within the finger. In this case, grains sediment on the bottom plate inside the finger in layers a few particle diameters deep. The depth of these layers is expected to be some function of $\Delta P$, but this piece of information is not accessible for these experiments. For the results shown in Fig. 10 for $b=1.15 \mathrm{~mm}$, only experiments where the fingers are completely empty of grains are included. For the oilgrain case, since it is an isodense suspension, such gravity effects are not an issue.

The fact that change in the initial packing density strongly alters the behavior of the mobility is a piece of evidence supporting a relation between the increasing mobility and an overall decreasing compaction by fluidization during the finger growth. At $\sim 62 \%$ initial packing fraction, the granular material is far beyond the jamming transition. It takes time to reach a completely unjammed state. If, however, the granular material is already initially close to the jamming transition, as for the samples produced for the air-grain case with a packing fraction $\sim 56 \%$, the paste flows readily as an effective fluid. The mobility is roughly constant during the progression of the finger [see insets in Figs. 9(a) and 9(c)].

The decompaction during finger propagation is rather modest for the oil-grain experiments, and mainly localized around finger tip positions $X_{f}<1 \mathrm{~cm}$ [see Fig. 10(a)]. This suggests that the decompaction alone cannot explain the increasing mobility. In this case, lubrication effects due to restructuring in terms of particle migration [44] toward the center of the cell gap might be an important factor.

For both systems, the mobilities are found to be independent of the applied $\Delta P$. Hence, we average over all the experimental runs with variable $\Delta P$, for the various cell thick- 

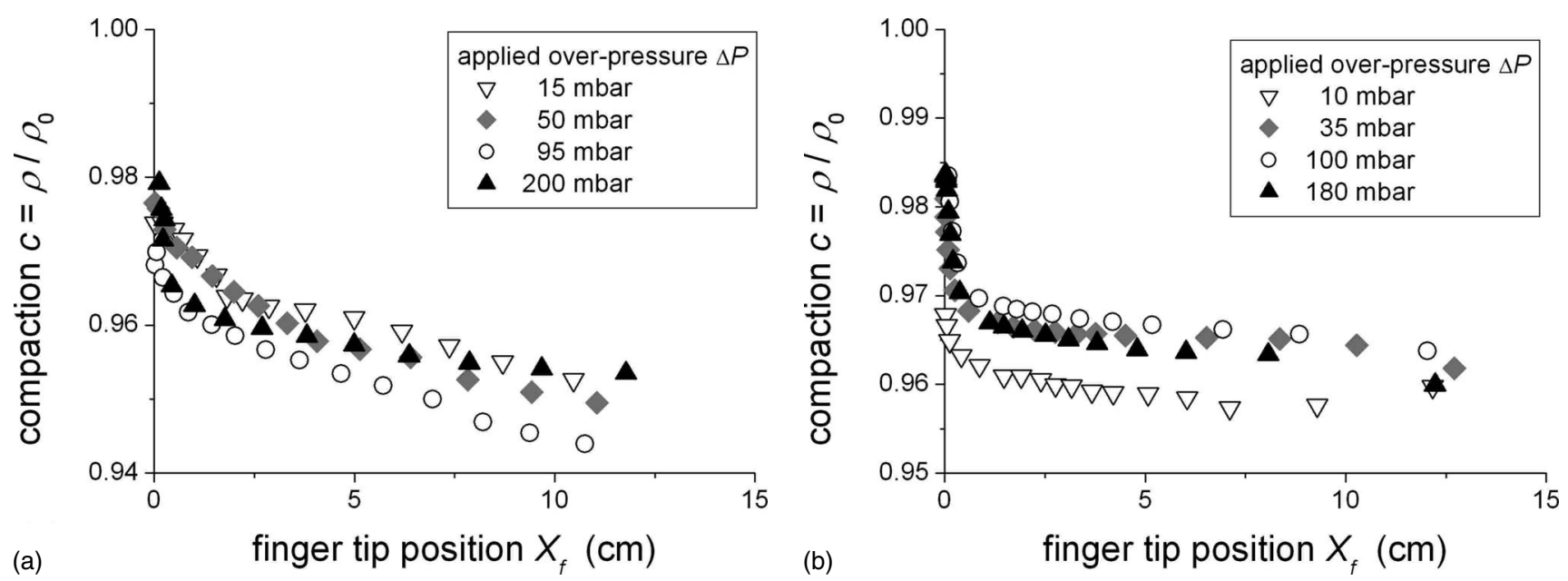

FIG. 10. Evolution of the compaction index [comparing average solid fractions, $\left.\rho(t) / \rho_{0}=A_{0} / A(t)\right]$ during finger propagation for (a) air-grain and (b) oil-grain systems.

nesses $b$. To be able to compare the magnitude of these averaged mobilities between the two systems, we scale $M$ with $d^{2} / \eta$, which is the typical mobility for flow of a fluid of viscosity $\eta$ through a pore of size $d$. This defines $M^{*}$ $=M /\left(d^{2} / \eta\right)$, where $d$ is the diameter of the grains, and $\eta$ the viscosity of the pure fluid (oil or air). $M^{*}$ is plotted as function of the finger tip position in Figs. 9(c) and 9(d), displaying, respectively, the air-grain and oil-grain experiments. We find that the mobilities compare favorably - they are roughly of the same order of magnitude for the two systems. For both air-grain and oil-grain experiments, there is a noticeable effect on the mobility from varying $b$. There is a tendency of a higher mobilization rate for larger $b$, given the increasing slopes from small to large $b$. During finger propagation the mobilities seem to saturate, indicating that there might be a transition to a steady state of the paste flow, characterized by a constant mobility at a given $b$. The experiments performed at an initially lower packing fraction and close to the jamming transition indeed show the existence of such a state [see inset in Fig. 9(c)]. To further investigate the transition from a regime where the mobility is evolving with the finger tip position to a stationary flow regime, a longer sample would be required.

For the air-grain system, the overall higher mobility for increasing cell thickness $b$ might be attributed to less effective friction between the fluidized granular material and its confining walls as $b$ is made larger, by considering friction mobilization in terms of a Janssen type of stress distribution [45]. If indeed lubrication is a key component in explaining the positively evolving mobility, the friction argument fails for the oil-grain situation.

We know from other Hele-Shaw types of studies involving viscous fluids that the mobility behaves as a quadratic function of the cell thickness $b$. For flow through a porous medium the mobility is independent of $b$. Given the geometry and aspect ratio of our experiments, we are not able to distinguish a coherent relation with respect to this parameter.

\section{Finger widths}

By direct visual comparison, the propagating fingers are found to appear wider for the air-grain system. Tip splitting is more evident for the oil-grain experiments, and common for smaller cell thickness. Typical finger structures are shown in the left column in Fig. 11.

We characterize the observed patterns in terms of the individual finger width $w_{i}$ as defined in Sec. IV A. For all the probed $\Delta P$ and cell thicknesses $b$, there is a weak linear increase of the individual finger width as function of the finger tip position, for both the air-grain and the oil-grain systems. We use this feature to obtain an average individual finger width. For a given $\Delta P$ and $b$, the averaging is performed for finger widths corresponding to finger tip positions within the interval $8<X_{f}<17 \mathrm{~cm}$.

A striking difference is observed when applying the two different fluids: for the air-grain case the finger width is a slightly increasing function of $\Delta P$, as shown in Fig. 11(b). In this case, the width is nonsensitive to variation of the cell thickness $b$. On the contrary, for the oil-grain system, the finger width is observed to be independent of $\Delta P$ and thus independent of the finger velocity. However, for the oil-grain system the finger width is a function of $b$ [see Fig. 11(d)]. Thus, the main parameter defining the finger width is the applied overpressure $\Delta P$ for the air-grain case, whereas for the oil-grain situation the cell thickness $b$ selects the finger width.

\section{CONCLUSION}

We have presented an experimental study of two cases concerning fluid injection into a granular medium saturated by the same fluid as the injected phase. The two systems have been described phenomenologically and quantitatively within the parameter space of the applied fluid overpressure $\Delta P$ and the thickness of the granular phase $b$.

The basic mechanism of fluidization is the same for the two cases. Since the granular packing is in a dense initial state, close to the random close packed configuration, the system is completely jammed. In order to transport grains, the packing must first dilate. Dilation is observed starting at the outer boundary facing the outlet. This dilation is referred to in terms of decompaction in the sense that we are able to 

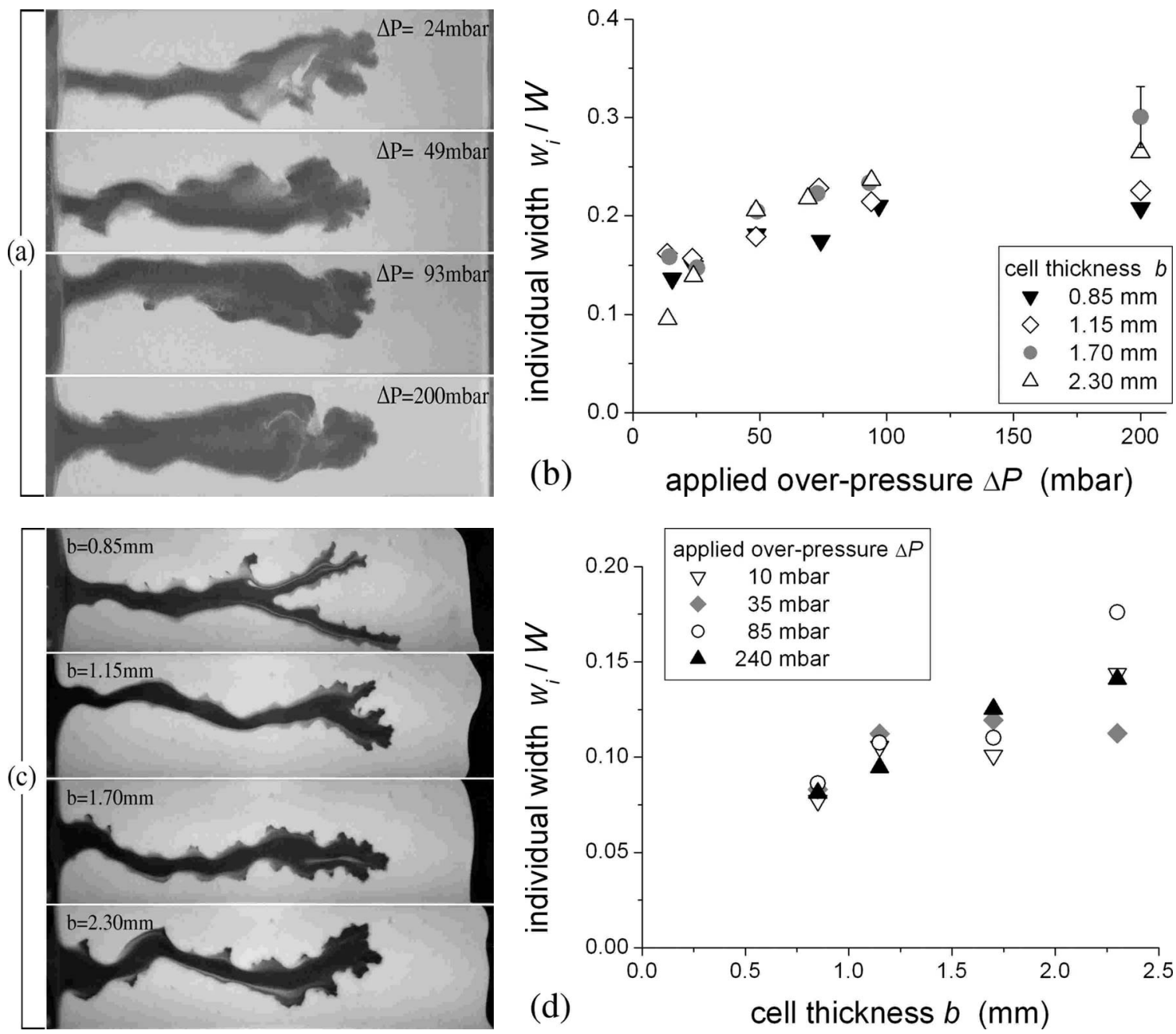

FIG. 11. (a), (c). Typical Patterns, respectively, for air-grain and oil-grain systems: for the presentation of air-grain results $b=1.15$ mm and $\Delta P$ is varied. For oil-grain systems the experiments are presented at a fixed $\Delta P \sim 55$ mbars but at different $b$. Right panels: Averaged individual finger width $w_{i}$, scaled on the cell width $W$ for (b) air-grain and (d) oil-grain systems. For the air-grain system, $w_{i} / W$ is a function of $\Delta P$. Each point represents an average over roughly 27 experiments. For the oil-grain system, $w_{i} / W$ is a function of $b$.

distinguish a zone with $\sim 3 \%$ decrease in solid fraction compared to the initial one. This decompaction zone evolves in time, propagating in the opposite direction of the effective fluid flow. During the passing of the decompaction front the granular packing is successively unjammed. In this way, the decompaction prepares the system for a very effective fluid and grain transport. When the decompaction front has reached the inner boundary, a localized zone characterized by a higher permeability connects the inlet side directly to the outlet side of the packing. Within this zone the particles are effectively transported along with the fluid, giving room for a fingerlike pattern emptied of grains to propagate through the decompacted medium.

Information on the unjamming decompaction process is retrieved by using an image subtraction technique, from which the temporal evolution of the decompaction area is found. The area measurements provide information on the average solid fraction of the decompacted region, which can be exploited to model the observed waiting time. The waiting time is the time it takes for the decompaction front to travel over the whole system length-hence representing the dura- tion for localized unjamming to occur over the entire length of the granular packing. The modeled waiting time compares favorably to both the respective experimental data sets. The waiting time found experimentally for the two systems compares very well when scaled with their fluid viscosity.

The emerging fingerlike patterns following the unjamming process of the granular phase are quite similar between the two systems. Geometrically, these fingers are quantified through their width. In both systems a slight widening of the fingers is observed during finger propagation. A striking difference on the finger width is observed as a result of injection of two different fluids. When the injection is made with air, the finger width is increasing as a function of $\Delta P$, but does not respond to varying the cell thickness $b$. However, when oil is injected into the oil-grain system the finger width is no longer a function of $\Delta P$ but increases instead with $b$. Furthermore, the fingers are typically wider in the air-grain case.

The fluidization process during finger propagation is described in terms of the mobility. Contrary to the constant mobilities measured in Hele-Shaw systems with strong 
analogies with the present study, i.e., two-fluid viscous fingering or fluid flow in fixed porous media, the mobility is increasing during finger propagation. This is the signature of a continuously more effective particle transport that might be attributed to a decreasing solid fraction during finger propagation, and perhaps also migration of the suspended particles toward the center of the cell gap in the oil-grain case. The mobilities of the two systems are of the same magnitude when scaled with respect to the fluid viscosity. For both systems the mobility of the flowing fluid-grain mixture is an increasing function of the cell thickness.

Evidently, experiments performed (air-grain system) at different initial packing fractions manifests the initial packing fraction as a crucial parameter for the overall dynamic behavior of the system. An initially loose granular matrix flows more like an effective fluid through the entire course of the experiment, recognized by a constant mobility during finger propagation. Prospected studies with a longer linear cell might reveal a transition from a regime characterized by an evolving mobility to such a stationary regime for the initially denser system.

\section{ACKNOWLEDGMENTS}

This work was supported by the Norwegian Research Council (NFR), CNRS through a PICS grant, and ANR through the ECOUPREF and TRIGGERLAND grants.
[1] H. S. Hele-Shaw, Nature (London) 58, 34 (1898).

[2] P. G. Saffman and G. I. Taylor, in Proc. R. Soc. London, Ser. A 245, 312 (1958).

[3] D. Bensimon, L. P. Kadanoff, S. Liang, B. I. Shraiman, and C. Tang, Rev. Mod. Phys. 58, 977 (1986).

[4] R. L. Chuoke, P. van Meurs, and C. van der Poel, Trans. Metall. Soc. AIME 216, 188 (1959).

[5] J. V. Maher, Phys. Rev. Lett. 54, 1498 (1985).

[6] D. Bonn, H. Kellay, M. Ben Amar, and J. Meunier, Phys. Rev. Lett. 75, 2132 (1995).

[7] G. Daccord, J. Nittmann, and H. E. Stanley, Phys. Rev. Lett. 56, 336 (1986).

[8] J. Nittman, G. Daccord, and H. E. Stanley, Nature (London) 314, 141 (1985).

[9] A. Lindner, P. Coussot, and D. Bonn, Phys. Rev. Lett. 85, 314 (2000).

[10] A. Lindner, D. Bonn, E. C. Poiré, M. Ben Amar, and J. Meunier, J. Fluid Mech. 462, 237 (2002).

[11] C. Chevalier, A. Lindner, and E. Clement, Phys. Rev. Lett. 99, 174501 (2007).

[12] H. Darcy, Les Fontaines Publiques de la Ville de Dijon (Dalmont, Paris, 1856).

[13] M. E. Cates, J. P. Wittmer, J.-P. Bouchaud, and P. Claudin, Phys. Rev. Lett. 81, 1841 (1998).

[14] A. L. Liu and S. Nagel, Nature (London) 396, 21 (1998).

[15] C. S. O’Hern, L. E. Silbert, A. J. Liu, and S. R. Nagel, Phys. Rev. E 68, 011306 (2003).

[16] T. S. Majmudar, M. Sperl, S. Luding, and R. P. Behringer, Phys. Rev. Lett. 98, 058001 (2007).

[17] S. F. Pinto, M. S. Couto, A. P. F. Atman, S. G. Alves, A. T. Bernardes, H. F. V. de Resende, and E. C. Souza, Phys. Rev. Lett. 99, 068001 (2007).

[18] K. Lu, E. E. Brodsky, and H. P. Kavehpour, Nat. Phys. 4, 404 (2008).

[19] Ø. Johnsen, R. Toussaint, K. J. Måløy, and E. G. Flekkøy, Phys. Rev. E 74, 011301 (2006).

[20] Ø. Johnsen, R. Toussaint, K. J. Måløy, E. G. Flekkøy, and J. Schmittbuhl, Phys. Rev. E 77, 011301 (2008).

[21] C. Chevalier, A. Lindner, M. Leroux, and E. Clément, J. NonNewtonian Fluid Mech. (2008) doi: 10.1016/ j.jnnfm.2008.07.007 (to be published).

[22] P. Mills, P. Cerasi, and S. Fautrat, Europhys. Lett. 29, 215 (1995).
[23] P. Cerasi and P. Mills, Phys. Rev. E 58, 6051 (1998).

[24] E. Lemaire, Y. O. M. Abdelhaye, J. Larue, R. Benoit, P. Levitz, and H. V. Damme, Fractals 1, 968 (1993).

[25] X. Cheng, L. Xu, A. Patterson, H. M. Jaeger, and S. R. Nagel, Nat. Phys. 4, 234 (2008).

[26] J. L. Vinningland, Ø. Johnsen, E. G. Flekkøy, R. Toussaint, and K. J. Måløy, Phys. Rev. E 76, 051306 (2007).

[27] J. L. Vinningland, Ø. Johnsen, E. G. Flekkøy, R. Toussaint, and K. J. Måløy, Phys. Rev. Lett. 99, 048001 (2007).

[28] E. G. Flekkøy, S. McNamara, K. J. Måløy, and D. Gendron, Phys. Rev. Lett. 87, 134302 (2001).

[29] D.-V. Anghel, M. Strauss, E. G. Flekkøy, S. McNamara, and K. J. Måløy, Phys. Rev. Lett. 97, 059902(E) (2006).

[30] X.-1. Wu, K. J. Måløy, A. Hansen, M. Ammi, and D. Bideau, Phys. Rev. Lett. 71, 1363 (1993).

[31] D. Gidaspow, Multiphase Flow and Fluidization (Academic Press, San Diego, CA, 1994).

[32] J. F. Davidson, Bubbles in Fluidized Beds (Kluwer Academic, New York, 1995), p. 197.

[33] J. F. Davidson and D. Harrison, Fluidization (Academic Press, New York, 1971).

[34] K. S. Lim, J. X. Zhu, and J. R. Grace, Int. J. Multiphase Flow 21, 141 (1995).

[35] C. Zeilstra, M. A. van der Hoef, and J. A. M. Kuipers, Phys. Rev. E 74, 010302(R) (2006).

[36] D. Lohse, R. Bergmann, R. Mikkelsen, C. Zeilstra, D. van der Meer, M. Versluis, K. van der Weele, M. van der Hoef, and H. Kuipers, Phys. Rev. Lett. 93, 198003 (2004).

[37] D. Lohse, R. Rauhé, R. Bergmann, and D. van der Meer, Nature (London) 432, 689 (2004).

[38] C. F. Wan and R. Fell, J. Geotech. Geoenviron. Eng. 130, 373 (2004).

[39] T. W. J. V. Asch, J. Buma, and L. P. H. V. Beek, Geomorphology 30, 25 (1999).

[40] T. Uchida, K. Kosugi, and T. Mizuyama, Hydrolog. Process. 15, 2151 (2001), and references therein.

[41] D. Karátson, J.-C. Thouret, I. Moriya, and A. Lomoschitz, Bull. Volcanol. (Heidelberg) 61, 174 (1999).

[42] G. D. Scott, Nature (London) 188, 908 (1960).

[43] P. C. Carman, Trans. Inst. Chem. Eng. 15, 150 (1937).

[44] D. Leighton and A. Acrivos, J. Fluid Mech. 181, 415 (1987).

[45] H. A. Janssen, Z. Ver. Dtsch. Ing. 39, 1045 (1892). 